\title{
Temperature Effects on Tensile and Compressive Mechanical Behaviors of C-S-H Structure via Atomic Simulation
}

\author{
Hao Xin, ${ }^{1}$ Weihui Lin, ${ }^{2}$ Jia Fu, ${ }^{3} \mathrm{Wu} \mathrm{Li}^{4}$ and Zhihua Wang ${ }^{1}$ \\ ${ }^{1}$ Shanxi Key Lab. of Material Strength \& Structural Impact, Taiyuan University of Technology, Taiyuan 030024, China \\ ${ }^{2}$ College of Mechanics, Taiyuan University of Technology, Taiyuan 030024, China \\ ${ }^{3}$ LGCGM, Institut National des Sciences Appliquées de Rennes, 35708 Rennes, France \\ ${ }^{4}$ Institute of Applied Mathematics, Taiyuan University of Technology, Taiyuan 030024, China \\ Correspondence should be addressed to Hao Xin; xinhao@tyut.edu.cn and Zhihua Wang; wangzh@tyut.edu.cn
}

Received 27 July 2017; Accepted 5 November 2017; Published 29 November 2017

Academic Editor: Ajayan Vinu

Copyright (c) 2017 Hao Xin et al. This is an open access article distributed under the Creative Commons Attribution License, which permits unrestricted use, distribution, and reproduction in any medium, provided the original work is properly cited.

\begin{abstract}
An atomic scale model of amorphous calcium silicate hydrate (C-S-H) with $\mathrm{Ca} / \mathrm{Si}$ ratio of 1.67 is constructed. Effects of temperature on mechanical properties of C-S-H structure under tensile and compressive loading in the layered direction are investigated via molecular dynamics simulations. Results from present simulations show that (1) the tensile strength and Young's modulus of C-S-H structure significantly decrease with the increase of the temperature; (2) the water layer plays an important role in the mechanical properties of C-S-H structure; (3) the compressive strength is stronger than tensile strength, which corresponds with the characteristic of cement paste.
\end{abstract}

\section{Introduction}

The hydration products of Portland cement are mainly calcium silicate hydrate (C-S-H) and calcium hydroxide $(\mathrm{CH})$, of which C-S-H accounts for about $60 \%-70 \%$ and $\mathrm{CH}$ about $20 \%$. C-S-H is the most important binding phase of cement paste, which has a great influence on the mechanical properties of the cement paste. The investigation of the C-S-H structure model and its mechanical properties for the C-S-H gel may help to improve the performance of concrete and to understand the constitutive relationship of the concrete, thus to design the high strength concrete with good durability. There have been many investigations of $\mathrm{C}-\mathrm{S}-\mathrm{H}$ since recent decades, though an accurate enough model is still under seeking so far to describe the atomic structure of C-S-H.

The atomic structure of C-S-H is commonly modeled from the tobermorite or jennite structure. Ye et al. [1] have built a long-range disordered and short-range ordered amorphous C-S-H structure by the annealing process based on Hamid's tobermorite model [2]. However, two basic characteristics, the calcium-to-silicon ratio $(\mathrm{Ca} / \mathrm{Si})$ and the density, of the amorphous C-S-H structure are incompatible with the real C-S-H. Murray et al. [3] have compared the mechanical properties of Hamid's $11 \AA$ tobermorite [2] and the possible C-S-H structure [4] by removal of the bridging silica tetrahedral of silicon chain in $11 \AA$ tobermorite, finding that the discontinuous silicon chain in C-S-H can lead to a decrease in the tensile strength. In fact, the nuclear magnetic resonance (NMR) experiment has confirmed that the siliconoxygen tetrahedron in C-S- $\mathrm{H}$ has a certain distribution of $Q_{n}$ : $Q_{0} \approx 10 \%, Q_{1} \approx 67 \%$, and $Q_{2} \approx 23 \%$ [5]. Besides, research by small-angle neutron scattering measurements also gave out the chemical component as $(\mathrm{CaO})_{1.7}\left(\mathrm{SiO}_{2}\right)\left(\mathrm{H}_{2} \mathrm{O}\right)_{1.8}$ and determined the $\mathrm{Ca} / \mathrm{Si}$ ratio and the density of $\mathrm{C}-\mathrm{S}-\mathrm{H}$ particles, which are separately 1.7 and $2.6 \mathrm{~g} / \mathrm{cm}^{3}[6]$.

Pellenq et al. [7] have proposed a cCSH model based on experimental data $[5,6]$, with the $\mathrm{Ca} / \mathrm{Si}$ ratio of 1.7 and the density of $2.45 \mathrm{~g} / \mathrm{cm}^{3}$. In accordance with the experimental data, Pellenq et al. [7] have also proposed a bottom-up atomic modeling method of the cCSH molecular structure, which can be described mainly as follows: the supercell of the anhydrous $11 \AA$ tobermorite [2] for the initial configuration is constructed without considering the presence of any $\mathrm{OH}$ groups; some of the silicon-oxygen tetrahedrons are deleted to obtain a defected C-S-H structure, where $Q_{0}=10 \%$, $Q_{1}=67 \%, Q_{2}=23 \%$, and $\mathrm{Ca} / \mathrm{Si}=1.65$, using the core-shell 
potential for relaxation at $0 \mathrm{~K}$; the water adsorption of the $\mathrm{C}$ $\mathrm{S}-\mathrm{H}$ model at $300 \mathrm{~K}$ with the Grand Canonical Monte Carlo (GCMC) is simulated, with a density of $2.56 \mathrm{~g} / \mathrm{cm}^{3}$, with further relaxation at $0 \mathrm{~K}$. The final model with the chemical composition of $(\mathrm{CaO})_{1.65}\left(\mathrm{SiO}_{2}\right)\left(\mathrm{H}_{2} \mathrm{O}\right)_{1.75}$ is obtained with a density of $2.45 \mathrm{~g} / \mathrm{cm}^{3}$, which has a good agreement with the neutron scattering experiments of $(\mathrm{CaO})_{1.7}\left(\mathrm{SiO}_{2}\right)\left(\mathrm{H}_{2} \mathrm{O}\right)_{1.8}$.

According to the modeling method of Pellenq et al., Hou et al. [8,9] have investigated the mechanical properties of $\mathrm{C}-\mathrm{S}-\mathrm{H}$ during the axial stretching process in three different directions using ClayFF [10] force field, as well as the influence of the contained water percentage on the mechanical properties of the C-S-H using the CSH-FF [11] force field. For Young's modulus of C-S-H, Constantinides and Ulm [12] have obtained Young's modulus of LD C-S-H and HD C-S$\mathrm{H}$ by nanoindentation experiment, which are $21.7 \mathrm{GPa}$ and 29.4 GPa, separately. Hou et al. [9] have also investigated the effects of water/calcium ratio $(\mathrm{W} / \mathrm{C}=0.0-1.0)$ on the mechanical properties of the layered C-S-H based on the CSH-FF force field. Their work shows that Young's modulus decreases from $67 \mathrm{GPa}$ to $47 \mathrm{GPa}$ and the maximum tensile strength decreases from 7.5 GPa to $3.8 \mathrm{GPa}$ with the increase in water.

However, in practical engineering, concrete is often exposed in a variety of environments, where certain parameters (temperature, pressure, and humidity) may have a greater influence on the mechanical properties of the C-S-H. For instance, the extreme temperature in Russia and Canada could be lower than $200 \mathrm{~K}$ (where local people still live) and some concrete structures in certain factories (such as nuclear power plants) may suffer as high temperature as $400 \mathrm{~K}$ or even higher. However, the influence of the temperature on Young's modulus of C-S-H structure on the nanometer scale has seldom been considered and investigated yet. In order to understand the macroscopic mechanical properties of concrete, it is necessary to understand the structure and behavior of C-S-H gel at the atomic level. In present work, we mainly focus on the influence of the temperature on mechanical properties of C-S-H structure under tensile/compressive loading. The stress-strain curve, Young's modulus, density, tensile strength, and tensile deformation process of C-S-H under different temperatures were analyzed.

\section{Numerical Simulation of C-S-H}

2.1. Model and Force Field. For the real amorphous C-S-H, the main features of the structure need to be considered, such as long-range disorder, short-range order, calcium silicon ratio $(\mathrm{Ca} / \mathrm{Si})$, the layered structure, $Q_{n}$ distribution, and density. The construction of the amorphous C-S-H model is referred to as Pellenq's cCSH model based on the experimental data $[5,6]$. The initial configuration is monoclinic as shown in Figures 1(a) and 1(b) showing how to make an orthogonal box conversion of the atomic structure before the simulation. The relative atomic position and model size remain unchanged after the box transformation.

Figure 2 records the main procedures of C-S-H model construction. First of all, the orthogonal supercell of Hamid's $11 \AA$ tobermorite is prepared and the presence of hydroxyl groups and water molecules are not considered. Then some parts of the $\mathrm{SiO}_{2}$ group in silica tetrahedron are deleted to satisfy $Q_{n}$ distribution and $\mathrm{Ca} / \mathrm{Si}$ ratio. Based on that, the intralayer calcium atoms are allowed to relax by energy minimization using ClayFF potential, followed by all the other atoms and the supercell dimensions. Grand Canonical Monte Carlo method [13] is used to simulate the water molecules adsorption using the same potential at room temperature. The final C-S-H with the chemical composition $(\mathrm{CaO})_{1.67}\left(\mathrm{SiO}_{2}\right)\left(\mathrm{H}_{2} \mathrm{O}\right)_{1.69}$, the density of $2.45 \mathrm{~g} / \mathrm{cm}^{3}$, and the reasonable $Q_{n}$ distribution $\left(Q_{0}=11.6 \%, Q_{1}=65.1 \%, Q_{2}=\right.$ $23.3 \%$ ) is obtained, which is in good agreement with the results from neutral scattering tests [6].

The ClayFF force field is suitable to simulate crystalline compounds containing water state and so forth [10]. ClayFF is also applied in current work to simulate the structure and investigate mechanical properties of the C-S-H model. SPC model is used for the water molecules [14, 15]. LAMMPS [16] program, a classical molecular dynamics (MD) code, is used to perform the MD simulation of C-S-H. In order to eliminate the border effect due to the size restriction of the system, the periodic boundary condition is set in the simulations. The parameters of ClayFF force field are detailed in [10]. In the present work, all atoms are classified into seven types: $\mathrm{Ca}$ (interlayer calcium), Cw (intralayer calcium), Si (silicon), $\mathrm{Ob}$ (bridging oxygen in silica chain), $\mathrm{O}$ (nonbridging oxygen in silica chain or calcium layers), Ow (oxygen in water molecule), and Hw (hydrogen in water molecule).

2.2. Simulation Procedure. The Lennard-Jones (12-6) potential type is employed to describe the interactions of the nonbonded atoms, which can be expressed as

$$
E_{i j}=4 \varepsilon_{i j}\left[\left(\frac{\sigma_{i j}}{r_{i j}}\right)^{12}-\left(\frac{\sigma_{i j}}{r_{i j}}\right)^{6}\right],
$$

where the cutoff radius of Van der Waals force is $8.0 \AA$. Meanwhile, parameters of $\varepsilon_{i j}$ and $\sigma_{i j}$ are estimated by using the arithmetic mixing rules as

$$
\begin{gathered}
\varepsilon_{i j}=\sqrt{\varepsilon_{i} \varepsilon_{j}}, \\
\sigma_{i j}=\frac{\sigma_{i}+\sigma_{j}}{2} .
\end{gathered}
$$

PPPM summation method [17] is used to calculate the long-range Coulomb interactions, and the calculation precision is set to 0.00001 . During the GCMC simulation, the temperature is set to $300 \mathrm{~K}$ and the chemical potential is set to zero, with the time step of $0.1 \mathrm{fs}$; after GCMC simulation, a $2.20 \mathrm{~nm} \times 2.18 \mathrm{~nm} \times 2.24 \mathrm{~nm} \mathrm{C}-\mathrm{S}-\mathrm{H}(\mathrm{C} / \mathrm{S}=1.67)$ model $(4 a \times$ $3 b \times 1 c)$ is obtained with 981 atoms and the density increases to $2.45 \mathrm{~g} / \mathrm{cm}^{3}$; then the box is relaxed by the NPT equilibrium condition about $25 \mathrm{ps}$, where the pressure is controlled to be zero in all $x, y$, and $z$ directions. The velocity Verlet method is used to integrate the classical equations of motion with a time step of $0.1 \mathrm{fs}$. A bigger C-S-H model is constructed by periodic extension in $x, y, z$ directions to $4.5 \times 4.5 \times 8.8 \mathrm{~nm}^{3}$ (15696 atoms in total) and then is relaxed for 20 ps using NPT ensembles in order to obtain a final stable system for MD simulation. 


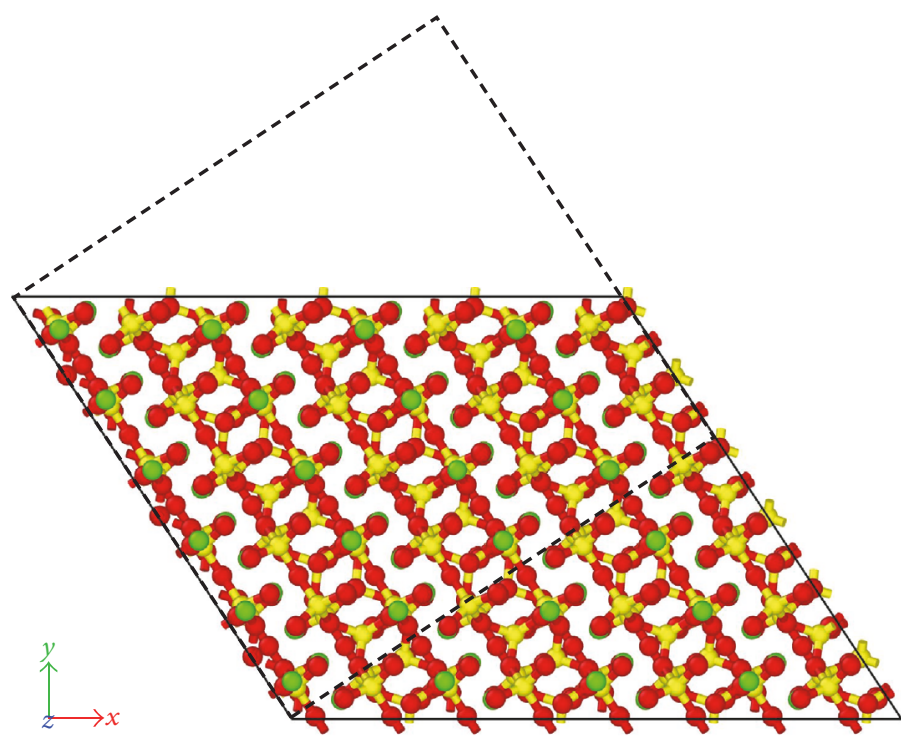

(a) Original box of a monoclinic cell

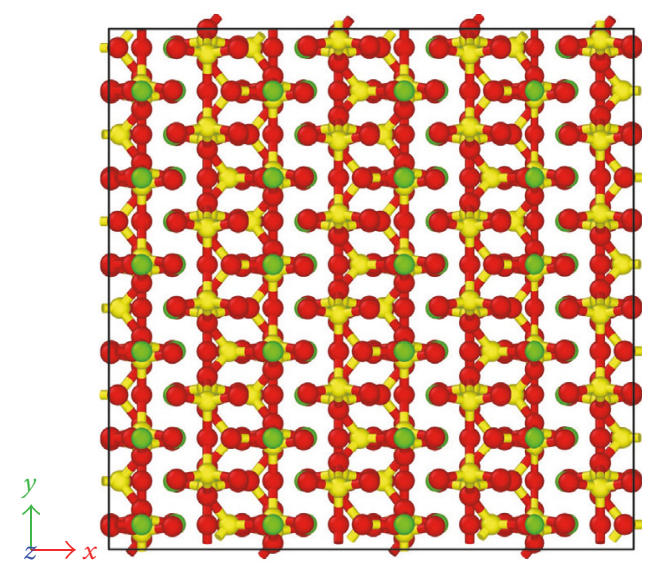

(b) Transformed box of a orthogonal cell

FIGURE 1: Schematic conversion diagram of simulation box in $x-y$ plane. (a) Initial $11 \AA$ tobermorite structure without water molecules and hydroxyl groups. Cell parameters: $a=26.76 \AA, b=22.17 \AA, c=22.77 \AA, \alpha=\beta=90^{\circ}, \gamma=123.49^{\circ}$. (b) Transformed box parameters: $a=22.32 \AA$, $b=22.17 \AA, c=22.77 \AA, \alpha=\beta=\gamma=90^{\circ}$.

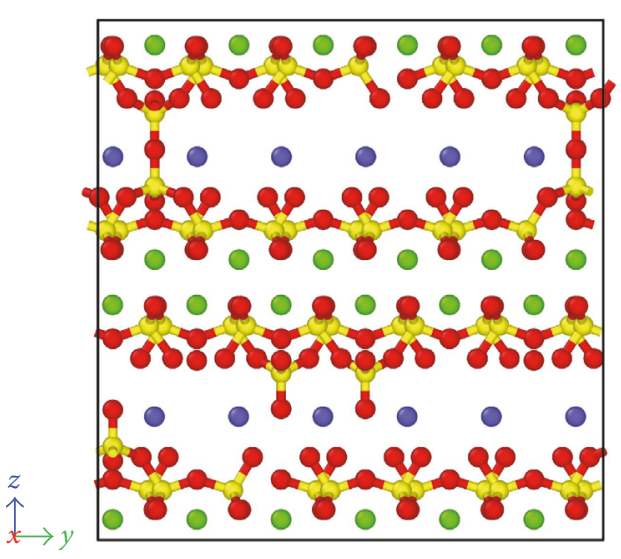

(a)

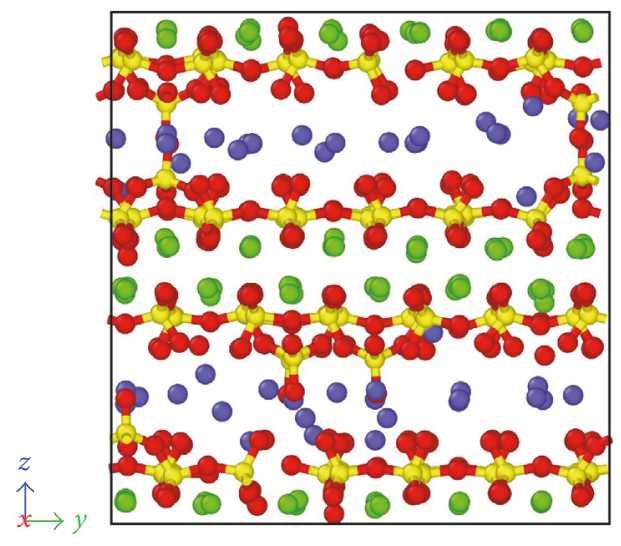

(c)

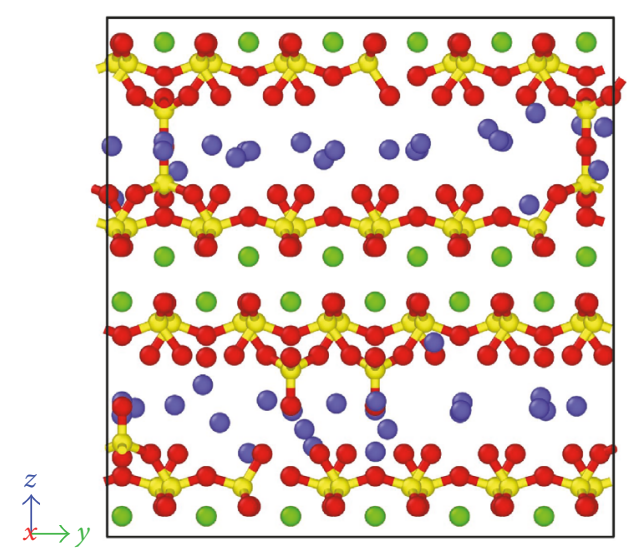

(b)

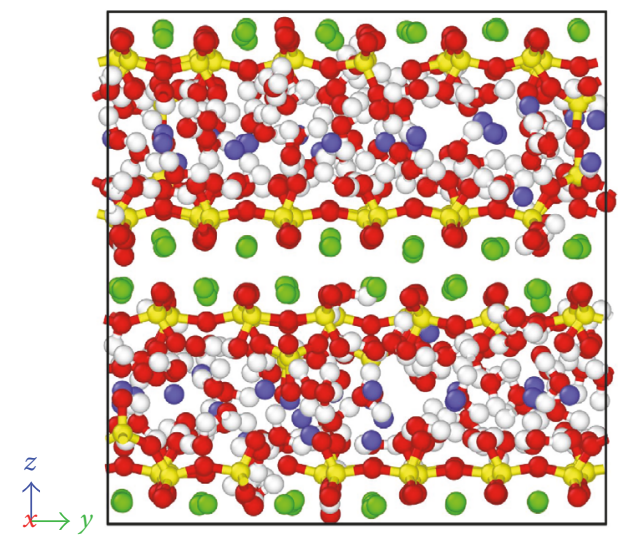

(d)

Figure 2: Construction process of C-S-H model (red ball: oxygen atom; green ball: interlayer calcium atom; blue ball: intralayer calcium; white ball: hydrogen atom; yellow tetrahedral: silicate tetrahedral): (a) remove some $\mathrm{SiO}_{2}$; (b) relax intralayer calcium atoms; (c) relax all the other atoms and the supercell dimensions; (d) adsorb water molecules. 


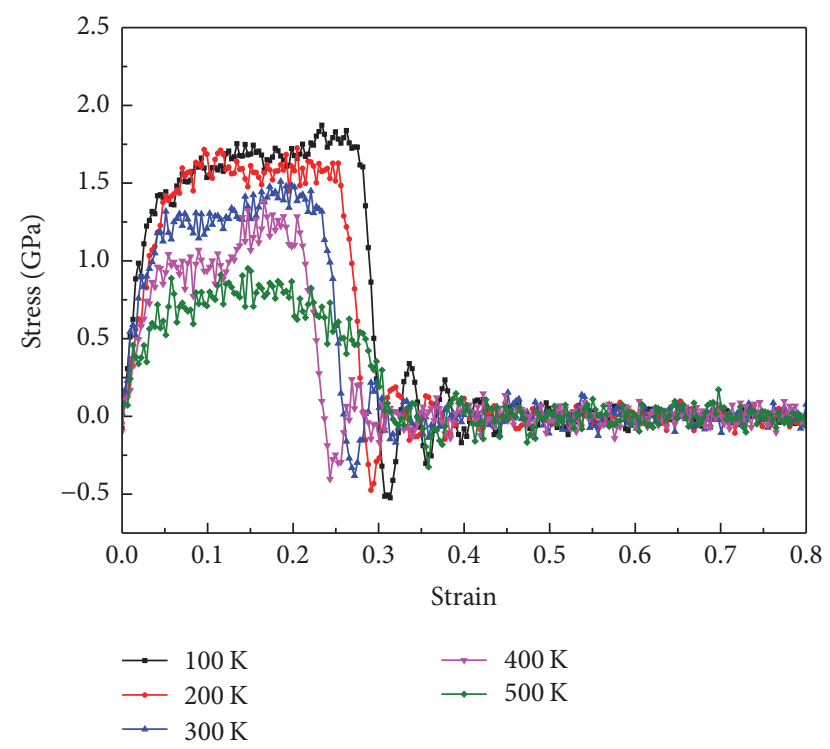

FIGURE 3: Stress-strain curves under uniaxial tension along the $z$ direction.

The tensile/compressive loading is imposed by changing the $z$-axis dimension of C-S-H with a strain rate of $0.08 / \mathrm{ps}$ under the NPT ensemble. The pressure in $z$ direction is not controlled during the loading process, while pressures in the other two directions remain zero. As the tensile strength in layered direction is the weakest as given by Hou et al. [8], this paper mainly focuses on the temperature effect on the behavior at layered direction ( $z$-direction) to further investigate the tensile/compressive mechanical properties of $\mathrm{C}-\mathrm{S}-\mathrm{H}$ gel at various temperatures.

\section{Results and Discussion}

In consideration of the potential application of concrete structure, as mentioned in the 1st section of this paper, uniaxial tensile and compressive strain rate were applied to the atomic structure of $\mathrm{C}-\mathrm{S}-\mathrm{H}$ gel at a temperature range from $100 \mathrm{~K}$ to $500 \mathrm{~K}$. The internal force on the atoms and deformation of the model were extracted and calculated for further analysis.

Figure 3 shows the stress-strain curves (layered direction) under uniaxial tension at different temperatures. A linear portion can be observed, and hence the elastic modulus can be ascertained. The elastic modulus of the C-S-H gel was computed by the slope of the linear portion of the tensile stress-strain curve. The data of the density, tensile strength, Young's modulus, and failure strain obtained from the uniaxial tension simulations of the C-S-H structure at different temperatures are shown in Table 1. Young's modulus in the present work is $27.3 \mathrm{GPa}$ at $300 \mathrm{~K}$, which is in good agreement with the results reported before [8]. The tensile strength gradually reduces from $1.84 \mathrm{GPa}$ to $0.91 \mathrm{GPa}$ with the increase of the temperature from $100 \mathrm{~K}$ to $500 \mathrm{~K}$ at Figure 3. Meanwhile, for the stress-strain curves at temperatures from $100 \mathrm{~K}$ to $400 \mathrm{~K}$, the stress increases rapidly to reach
TABLE 1: Density and mechanical properties under uniaxial tension.

\begin{tabular}{lccccc}
\hline & $100 \mathrm{~K}$ & $200 \mathrm{~K}$ & $300 \mathrm{~K}$ & $400 \mathrm{~K}$ & $500 \mathrm{~K}$ \\
\hline Density $\left(\mathrm{g} / \mathrm{cm}^{3}\right)$ & 2.46 & 2.42 & 2.39 & 2.38 & 2.33 \\
Tensile strength $(\mathrm{GPa})$ & 1.84 & 1.73 & 1.51 & 1.38 & 0.91 \\
Young's modulus $(\mathrm{GPa})$ & 33.3 & 28.4 & 27.3 & 21.2 & 14.4 \\
Failure strain & 0.30 & 0.28 & 0.26 & 0.24 & 0.20 \\
\hline
\end{tabular}

the elastic limit, then the simulated structures undergo a yielding stage followed by a strain-hardening stage. After the stress increases to the ultimate value, each system exhibits a characteristic of brittle fracture. However, the curve of $500 \mathrm{~K}$ is different from the others. When the temperature is up to $500 \mathrm{~K}$, there is no longer the yield plateau or the strainhardening stage.

It is clear from Table 1 that the density decreases with the increase of the temperature, from $2.46 \mathrm{~g} / \mathrm{cm}^{3}$ at $100 \mathrm{~K}$ to $2.33 \mathrm{~g} / \mathrm{cm}^{3}$ at $500 \mathrm{~K}$, which indicates that there is little temperature effect on the atomic structure of $\mathrm{C}-\mathrm{S}-\mathrm{H}$ within the range of $100 \mathrm{~K}-500 \mathrm{~K}$. Besides, the tensile strength of present C-S-H structure (with water/calcium ratio of 0.92 ) also decreases from $1.84 \mathrm{GPa}$ to $0.91 \mathrm{GPa}$, which is close to the results of the model with water/calcium ratio 0.95 by Hou et al. [9]. Moreover, Young's modulus at $300 \mathrm{~K}$ is about $27.3 \mathrm{GPa}$, which is quite close to the nanoindentation experiment result 29.4 GPa by Constantinides and Ulm [12] and the simulation result 26.1 GPa obtained by Hou et al. [8]. It could be found that the temperature changes have great effects on Young's modulus, which decreases from $33.3 \mathrm{GPa}$ to $14.4 \mathrm{GPa}$ with the increase in temperature.

Figure 4 shows how the damage occurred in the C-S-H structure during the stretching process at different temperatures, which indicates a clearly temperature dependence of the failure mode. It can be clearly observed that the water layers are gradually separated with the increase of the tensional strain, subsequently leading to the cracks formed in C-S-H. In fact, it is due to these cracks of the water layers that the tensile strength of C-S-H significantly decreases in the layered direction. In other words, the intralayer water molecules are the weakest parts of the C-S-H gel, which makes the whole structure (at atomic level or macroscopically) brittle and weak.

The behavior of $\mathrm{C}-\mathrm{S}-\mathrm{H}$ under uniaxial compression was also simulated and the compressive stress-strain curves are presented in Figure 5(a). A good linear portion can be observed at the beginning stage of the curves though each curve is fluctuating consistently due to the statistical attributes of MD data. And Figure 5(b) displays the linear fit value of the compressive stress at different temperatures. The linear slope decreases clearly with the increase of the temperature. The simulated compressive strength, Young's modulus, and peak strain of C-S-H are listed in Table 2. Young's modulus obviously decreases with the increase in temperature and there is almost a reduction trend of the compressive carrying capacities. However, the strength and peak strain at $100 \mathrm{~K}$ are not the greatest at all. Meanwhile, it can be found in Figure 5(a) that the compressive stressstrain curves do not exhibit yield plateau in all the simulated 


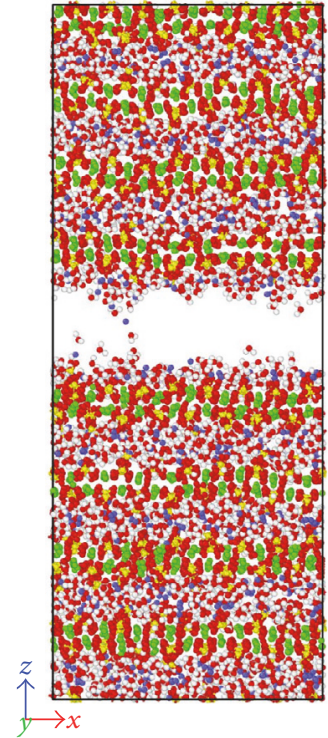

(a)

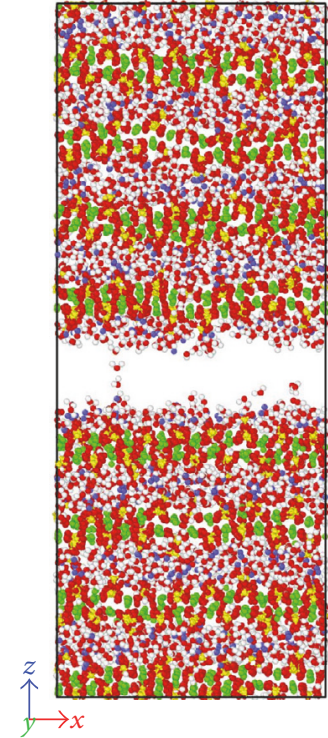

(b)

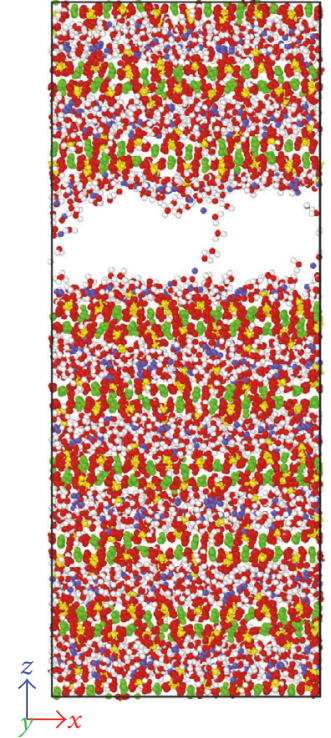

(c)

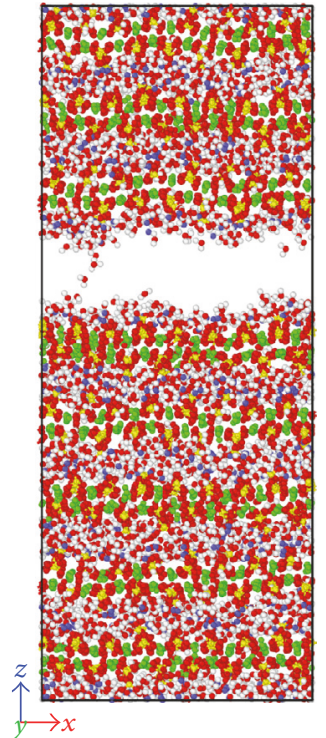

(d)

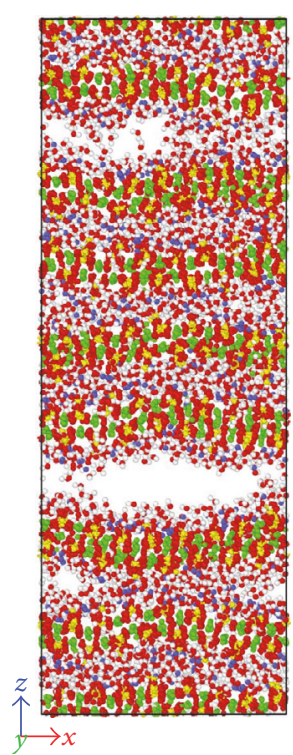

(e)

Figure 4: The tensile deformation at different temperatures: (a) $100 \mathrm{~K}$; (b) $200 \mathrm{~K}$; (c) $300 \mathrm{~K}$; (d) $400 \mathrm{~K}$; (e) $500 \mathrm{~K}$.

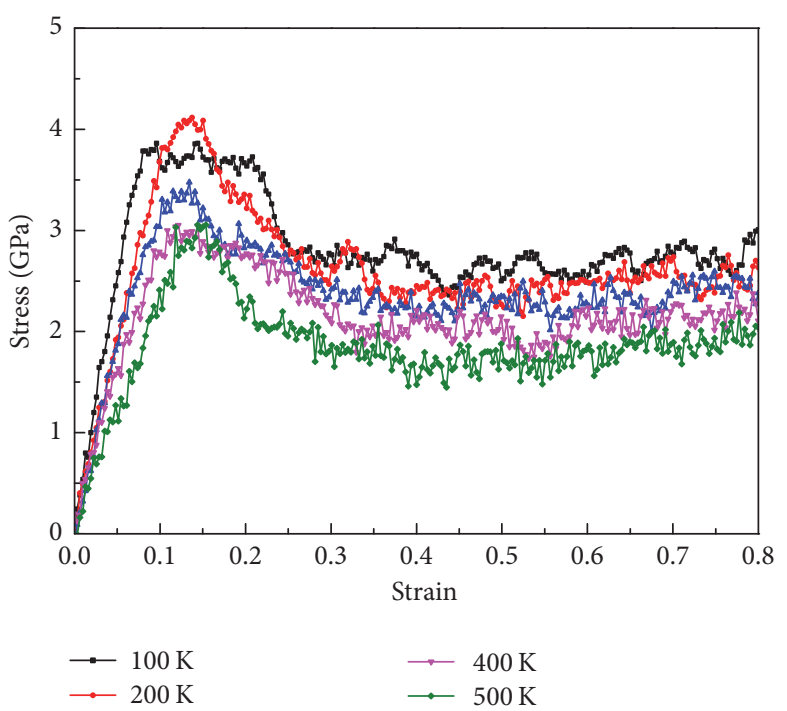

(a)

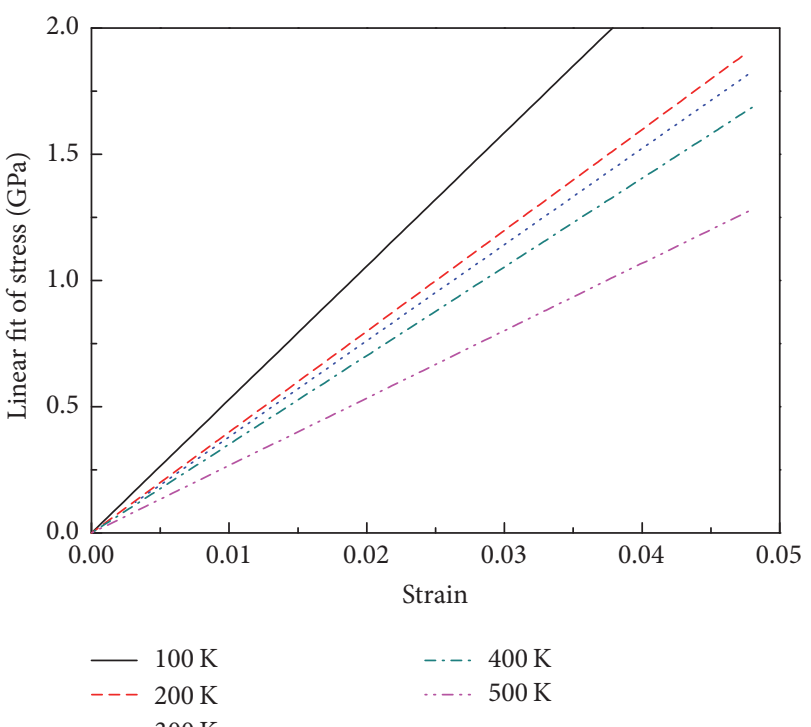

(b)

FiguRE 5: Stress-strain curves under uniaxial compression along the $z$ direction.

cases except at $100 \mathrm{~K}$. It seems that extremely low temperature does not make C-S-H stronger and brittle but makes it lose bearing capacities prematurely and come through a yielding stage. At $200 \mathrm{~K}-500 \mathrm{~K}$, the compressive stress increases to the ultimate value and then the structure immediately fails, which is consistent with the brittle characteristic of cement and concrete in the usual impression. All those imply a great influence of temperature on the compressive performance of the C-S-H gel.

Comparing the cases of tension and compression, the compressive strength of the C-S-H gel is much larger than the
TABLE 2: Mechanical properties under uniaxial compression.

\begin{tabular}{lccccc}
\hline & $100 \mathrm{~K}$ & $200 \mathrm{~K}$ & $300 \mathrm{~K}$ & $400 \mathrm{~K}$ & $500 \mathrm{~K}$ \\
\hline Compressive strength $(\mathrm{GPa})$ & 3.86 & 4.12 & 3.47 & 3.05 & 3.05 \\
Young's modulus $(\mathrm{GPa})$ & 44.8 & 37.6 & 34.0 & 29.8 & 23.5 \\
Peak strain & 0.096 & 0.138 & 0.134 & 0.122 & 0.121 \\
\hline
\end{tabular}

tensile one at certain temperature. Similar situation happens at the macrolevel because it is universally known that the cement paste is strong in compression and weak in tension. 
Therefore, the discrepancy between the compressive and tensile behavior of C-S-H gel at the nanolevel also gives a kind of atomic insight into the tensile weakness of cement paste.

\section{Conclusion}

The molecular structure, mechanical properties, and temperature effect of C-S-H gel at the atomic level were investigated via $\mathrm{MD}$ simulations. The uniaxial loading processes in the layered direction of C-S-H gel were simulated at different temperatures and the relative stress-strain curves and density were analyzed. Main results are as follows:

(1) With the increase in temperature, the tensile strength of C-S-H decreases significantly.

(2) Young's modulus reduces with the increase in temperature, from $33.3 \mathrm{GPa}$ to $14.4 \mathrm{GPa}$ under tension and from $44.8 \mathrm{GPa}$ to $23.5 \mathrm{GPa}$ under compression.

(3) The water layer is an important factor affecting the mechanical properties and deformation performance of C-S-H.

(4) C-S-H becomes stronger and more brittle under compression as the temperature increases, but extremely low temperature does not make C-S-H stronger but makes it lose bearing capacities prematurely.

(5) C-S-H gel shows a strong compressive strength in compression and a weak tensional strength, which helps to understand the characteristic of cement paste better at the macrolevel.

\section{Conflicts of Interest}

The authors declare that they have no conflicts of interest.

\section{Acknowledgments}

The authors greatly acknowledge the financial support for this work provided by the National Science Foundation of China (11390362, 11402164), Special Program for Applied Research on Super Computation of the NSFC-Guangdong Joint Fund (the second phase, Grant no. U1501501), and Science Foundation of Shanxi Province (2015021024).

\section{References}

[1] J. Ye, W. Zhang, H. Wang, Y. Wang, and J. Zhang, "Structure of calcium silicate hydrate Ca4Si6O14 $(\mathrm{OH}) 4 \cdot 2 \mathrm{H} 2 \mathrm{O}$ simulated by the molecular dynamics," Kuei Suan Jen Hsueh Pao/Journal of the Chinese Ceramic Society, vol. 38, no. 12, pp. 2346-2352, 2010.

[2] S. A. Hamid, "The crystal structure of the $11 \AA$ natural tobermorite Ca2.25[Si3O7.5(OH)1.5]1H2O," Zeitschrift fur Kristallographie - New Crystal Structures, vol. 154, no. 3-4, pp. 189-198, 1981.

[3] S. J. Murray, V. J. Subramani, R. P. Selvam, and K. D. Hall, "Molecular dynamics to understand the mechanical behavior of cement paste," Transportation Research Record, no. 2142, pp. 75-82, 2010.
[4] V. J. Subramani, S. Murray, and R. P. Selvamet, "Atomic Structure of Calcium Silicate Hydrates Using Molecular Mechanics," Research Board 88th Annual Meeting, 2009.

[5] J. B. d'Espinose de la Caillerie and N. Lequeux, "Lecture on the structure of CSH," in AFm and AFt phases, Physique, Chimie et Mécanique des Matériaux Cimentaire, ed Ecole ATILH-CNRS, 2008.

[6] A. J. Allen, J. J. Thomas, and H. M. Jennings, "Composition and density of nanoscale calcium-silicate-hydrate in cement," Nature Materials, vol. 6, no. 4, pp. 311-316, 2007.

[7] R. J.-M. Pellenq, A. Kushima, R. Shahsavari et al., "A realistic molecular model of cement hydrates," Proceedings of the National Acadamy of Sciences of the United States of America, vol. 106, no. 38, pp. 16102-16107, 2009.

[8] D. Hou, Y. Zhu, Y. Lu, and Z. Li, "Mechanical properties of calcium silicate hydrate $(\mathrm{CSH})$ at nano-scale: A molecular dynamics study," Materials Chemistry Physics, vol. 146, no. 3, pp. 503-511, 2004.

[9] D. Hou, H. Ma, Y. Zhu, and Z. Li, "Calcium silicate hydrate from dry to saturated state: Structure, dynamics and mechanical properties," Acta Materialia, vol. 67, pp. 81-94, 2014.

[10] R. T. Cygan, J.-J. Liang, and A. G. Kalinichev, "Molecular models of hydroxide, oxyhydroxide, and clay phases and the development of a general force field," The Journal of Physical Chemistry B, vol. 108, no. 4, pp. 1255-1266, 2004.

[11] R. Shahsavari, R. J.-M. Pellenq, and F.-J. Ulm, "Empirical force fields for complex hydrated calcio-silicate layered materials," Physical Chemistry Chemical Physics, vol. 13, no. 3, pp.1002-1011, 2011.

[12] G. Constantinides and F. J. Ulm, "The effect of two types of C$\mathrm{S}-\mathrm{H}$ on the elasticity of cement-based materials: results from nanoindentation and micromechanical modeling," Cement and Concrete Research, vol. 34, no. 1, pp. 67-80, 2004.

[13] J. Puibasset and R. J.-M. Pellenq, "Water adsorption on hydrophilic mesoporous and plane silica substrates: A grand canonical Monte Carlo simulation study," The Journal of Chemical Physics, vol. 118, no. 12, pp. 5613-5622, 2003.

[14] H. J. C. Berendsen, J. R. Grigera, and T. P. Straatsma, "The missing term in effective pair potentials," The Journal of Physical Chemistry C, vol. 91, no. 24, pp. 6269-6271, 1987.

[15] Q. Ji, R. J.-M. Pellenq, and K. J. Van Vliet, "Comparison of computational water models for simulation of calcium-silicatehydrate," Computational Materials Science, vol. 53, no. 1, pp. 234-240, 2012.

[16] S. Plimpton, "Fast parallel algorithms for short-range molecular dynamics," Journal of Computational Physics, vol. 117, no. 1, pp. 1-19, 1995.

[17] S. J. Plimpton, R. Pollack, and M. Stevens, Proceedings of the Eighth SIAM Conference on Parallel Processing for Scientific Computing, Minneapolis, Minnesota, Minn, USA, 1997. 

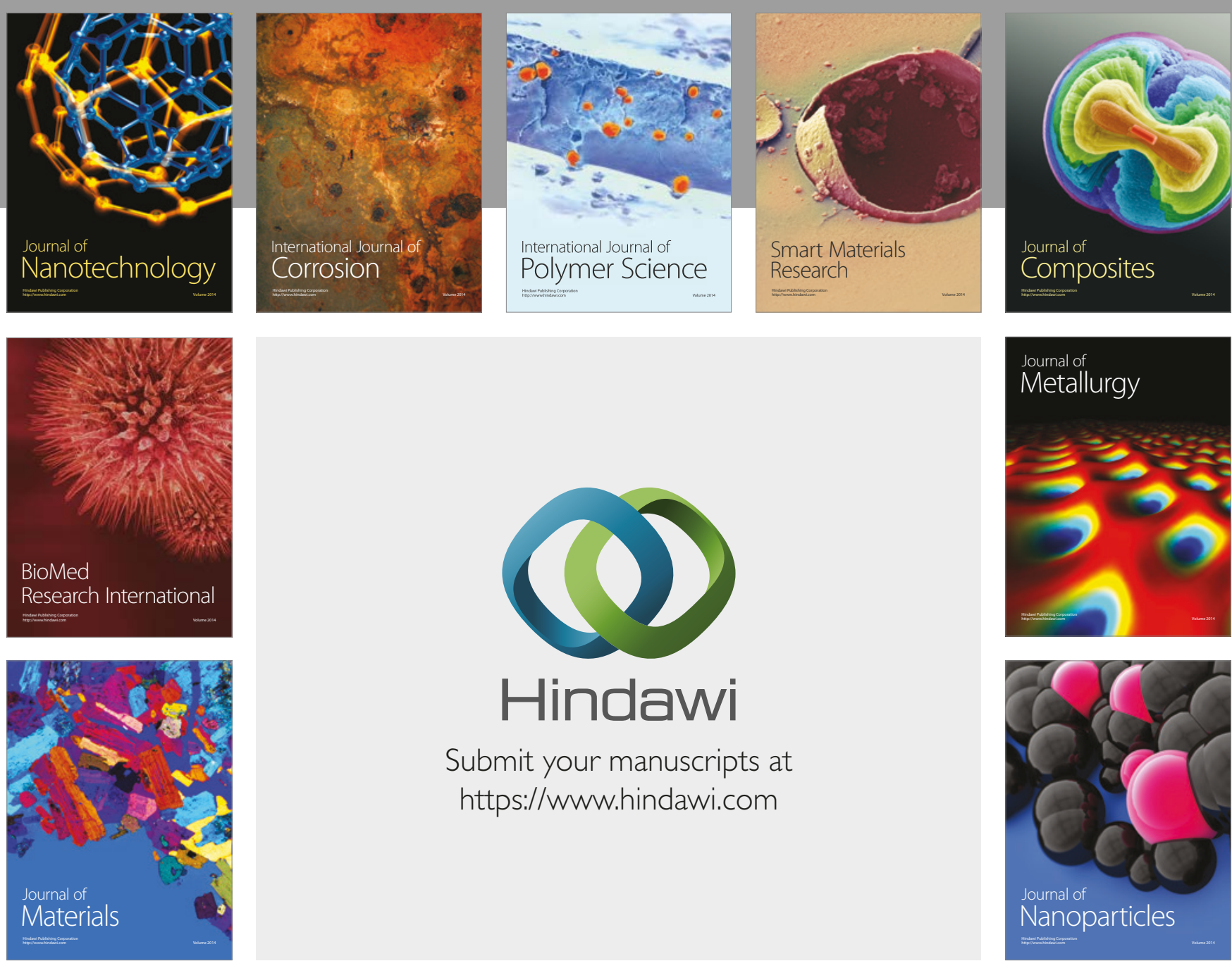

\section{Hindawi}

Submit your manuscripts at

https://www.hindawi.com
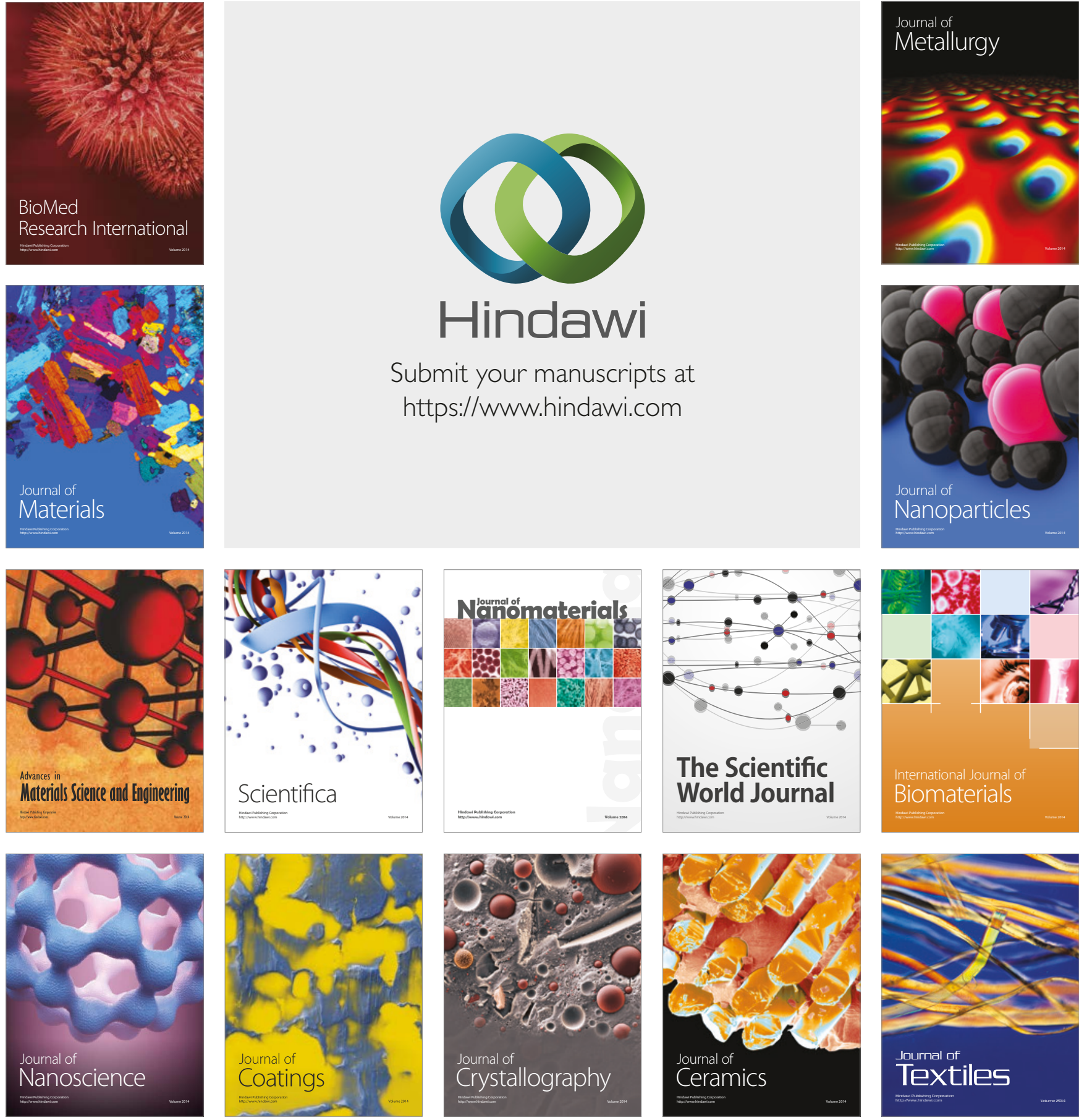

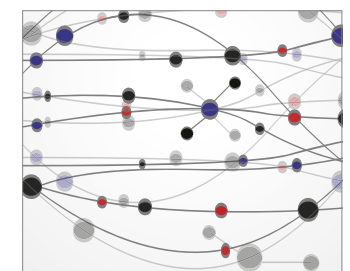

The Scientific World Journal
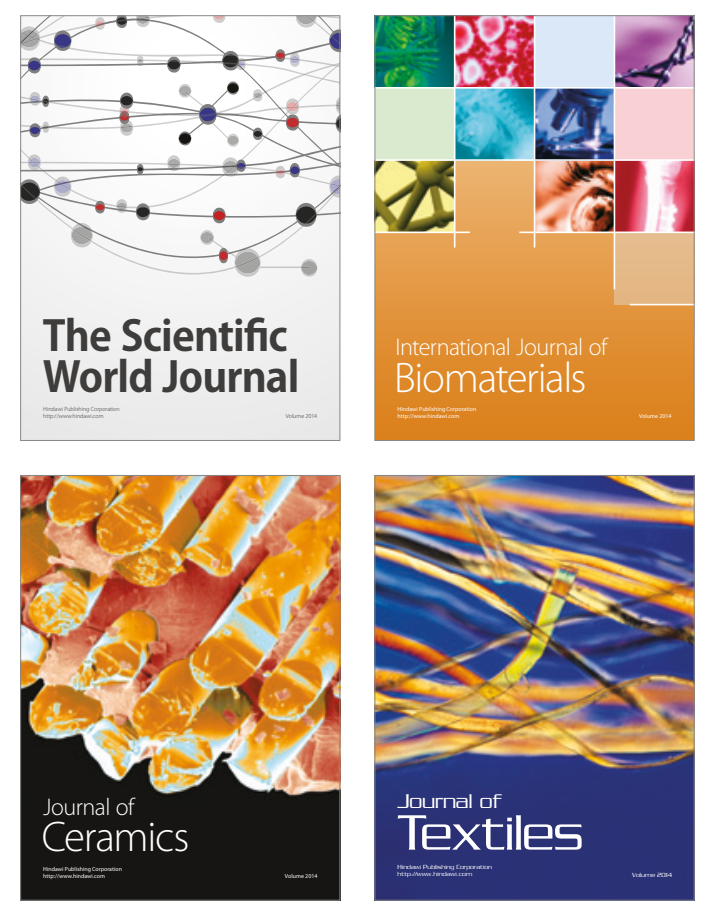